\title{
TAVI for Pure Non-calcified Aortic Regurgitation Using a Self-Expandable Transcatheter Heart Valve
}

Yvonne Schneeberger $^{1 *}$, Moritz Seiffert ${ }^{2}$, Andreas Schaefer $^{1}$, Oliver D. Bhadra ${ }^{1}$, Niklas Schofer ${ }^{2}$, Simon Pecha ${ }^{1}$, Dirk Westermann ${ }^{2}$, Stefan Blankenberg ${ }^{2}$, Hermann Reichenspurner ${ }^{1}$ and Lenard Conradi ${ }^{1}$

${ }^{1}$ Department of Cardiovascular Surgery, University Heart and Vascular Center Hamburg, Hamburg, Germany, ${ }^{2}$ Department of Cardiology, University Heart and Vascular Center Hamburg, Hamburg, Germany

\section{OPEN ACCESS}

Edited by:

Peter Zilla,

University of Cape Town, South Africa

Reviewed by:

Gabriele Tamagnini,

Villa Torri Hospital, Italy

Paul Human,

University of Cape Town, South Africa

*Correspondence:

Yvonne Schneeberger

y.schneeberger@uke.de

Specialty section: This article was submitted to

Heart Valve Disease,

a section of the journal

Frontiers in Cardiovascular Medicine

Received: 18 July 2021 Accepted: 08 December 2021 Published: 25 January 2022

Citation:

Schneeberger $Y$, Seiffert $M$, Schaefer A, Bhadra OD, Schofer N,

Pecha S, Westermann D,

Blankenberg S, Reichenspurner $\mathrm{H}$ and

Conradi L (2022) TAVI for Pure

Non-calcified Aortic Regurgitation

Using a Self-Expandable

Transcatheter Heart Valve.

Front. Cardiovasc. Med. 8:743579.

doi: 10.3389/fcrm.2021.743579
Objectives: Transcatheter aortic valve implantation (TAVI) is routinely performed in patients with severe aortic stenosis (AS). For patients with pure non-calcified aortic regurgitation (AR) who are not suitable for open heart surgery no clear recommendations exist and use of TAVI has been largely off-label. We herein report a series of patients treated with the self-expandable AcurateNeo and Neo2 (Boston Scientific Co., Marlborough, MS, USA) transcatheter heart valve (THV) for pure AR.

Methods: Between 05/2017 and 03/2021, 9 patients (88.8\% female, $74.4 \pm 7.1$ years, logEuroSCORE II $5.5 \pm 3.6 \%$, STS PROM $6.2 \pm 3.0 \%$ ) received transfemoral (TF) TAVI for pure non-calcified AR following an adjusted valve sizing algorithm. Data were retrospectively analyzed according to updated Valve Academic Research Consortium (VARC-2) definitions.

Results: Device success was $100 \%$. Early safety was $77.7 \%$ (7/10), due to two (22.2\%) cases of acute kidney injury. Thirty-day mortality was $0 \%$, in seven $(77.7 \%)$ patients no or trace paravalvular leakage (PVL) was seen and mild PVL in two (22.2\%) patients at 30-day follow-up. No permanent pacemaker (PPM) was required during 30-day follow-up.

Conclusion: In this series of selected patients using the Acurate neo THV for pure non-calcified AR, safety and efficacy were demonstrated. Thirty-day mortality as well as PPM implantation and PVL rates showed excellent results in this high-risk patient cohort. These results will have to be confirmed in larger patient cohorts.

Keywords: transcatheter aortic valve implantation, aortic valve, aortic regurgitation, self- expanding, transcatheter heart valve

\section{INTRODUCTION}

Transcatheter aortic valve implantation (TAVI) is routinely performed in patients with severe aortic stenosis (AS) at intermediate or high risk for surgical aortic valve replacement (SAVR), when anatomical conditions for an interventional approach are adequate (1-3). Correspondingly, TAVI has been incorporated in international guidelines $(4,5)$. While extension of TAVI to low risk patients remains controversial, mainly due to a higher risk of postprocedural permanent pacemaker (PPM) implantation, residual paravalvular leakage (PVL) and lack of long-term durability data as 
shown in registry analyses (6-8), evolution of transcatheter heart valves (THV) and corresponding delivery systems is continuing. Besides liberalization of TAVI indications, off-label use of THV for varying aortic valve diseases has been described, with broader clinical application in TAVI for pure non-calcified AR. Traditionally, pure AR is considered a contraindication for TAVI, since absence of aortic valve calcification can lead to insufficient anchoring of the stent frame with possible consecutive valve embolization or relevant PVL (9). However, patients with AR and high comorbidity burden may not be eligible for SAVR and the only THV certificated for AR due to a unique anchoring mechanism (JenaValve) has just very recently been commercially approved and is clinically not widely available (10). Patients with untreated severe AR and a left ventricular ejection fraction of $<30 \%$ have an annular mortality of up to $20 \%$ and only few of these patients undergo SAVR. Since AR prevalence increases with age, an increasing number of patients with AR in need for TAVI can be anticipated (11). The most frequently utilized THV for treatment of AR are reported to be the self-expandable (SE) CoreValve EvolutR (Medtronic PLC., Minneapolis, MN, US) (12), the SE Acurate neo (Boston Scientific Co., Marlborough, MS, US) (13) and balloon-expandable (BE) THV of the Sapien family (Edwards Lifesciences Inc., Irvine, CA, US) (14). Since particular design features of the Acurate neo THV (distal stabilization arches and upper/lower stent crown inner/outer pericardium skirts) have the potential to protect against valve embolization and residual PVL in non-calcified aortic valves it has become our default THV for treatment of pure AR in patients with a prohibitive risk for SAVR. We herein present our experience with this THV platform for treatment of pure AR with a special emphasis on preprocedural planning and intraprocedural considerations.

\section{MATERIALS AND METHODS}

\section{Patients}

Between 05/2017 and 03/2021, 9 consecutive patients received transfemoral (TF) TAVI using the Acurate neo and neo2 THV for pure non-calcified AR following an adjusted valve sizing algorithm. Assessment of prohibitive risk for SAVR [3/9 patients with previous cardiac surgery, 4/9 due to age and comorbidities, $1 / 9$ due to previous left ventricular assist device (LVAD) implantation and $1 / 9$ due to malignant disease] and allocation to TAVI followed current international recommendations (4, 5) after consensus of the local dedicated heart team. Written informed consent was obtained from all patients. The study was approved by the local institutional review board.

Abbreviations: AR, Aortic regurgitation; AS, Aortic stenosis; BE, Balloonexpandable; LVAD, Left ventricular assist device; PPM, Permanent pacemaker; PVL, Paravalvular leakage; RVP, Rapid ventricular pacing; SAVR, Surgical aortic valve replacement; SE, Self-expandable; TAVI, Transcatheter aortic valve implantation; TEE, Transesophageal echocardiography; TF, Transfemoral; THV, Transcatheter heart valve.

\section{Diagnostic Work-Up, Study Procedure, and Valve Sizing Algorithm}

The preprocedural diagnostic work-up followed institutional standards and was described before (15): By routine, all patients received preoperative transthoracic and/or transesophageal echocardiography (TEE), a contrast-enhanced, electrocardiogram-gated MSCT for calculation for native aortic annulus dimensions and determination of adequate THV size as well as assessment of aortic root anatomy and morphology with the 3mensio Medical Imaging Software (3mensio, Medical Imaging, Bilthoven, Netherlands). Valve sizing followed an adjusted algorithm proposed by Kim et al. for aortic valve stenosis (16) with additional oversizing equivalent to 10.7 $\pm 2.7 \%$ of the THV diameter when compared to the native annulus diameter in this series (see Table 1). As a crude measure, an effective perimeter-derived annular diameter of $25.5 \mathrm{~mm}$ was considered the absolute technical maximum even though secondary measures such as annular eccentricity and diameter of the left ventricular outflow tract were also considered and may have contraindicated procedures below this value.

First line approach for all procedures was local anesthesia and/or analgosedation. All procedures were performed in a specially equipped hybrid operating suite by a dedicated team of cardiologists, cardiac surgeons and anesthesiologists. The first step of THV deployment was conducted on the beating heart using fast pacing while, during the second deployment step, rapid ventricular pacing (RVP) was used to ensure stable THV positioning. In the LVAD patient, the guidewire was placed adjacent to the LVAD inflow by fluoroscopy and TEE guidance and LVAD flow was minimized during THV deployment to avoid ventricular migration. THV function was assessed by invasive measurements of hemodynamics, aortic root angiography, and TTE.

\section{Transcatheter Heart Valve}

The Boston Scientific Acurate neo (Boston Scientific, Marlborough, MA, USA) THV has a SE nitinol frame carrying porcine pericardial leaflets in a supraannular position (Figure 1). The most important difference to other self-expanding platforms is the top-down deployment with minimal protrusion of the stent toward the left ventricular outflow tract. In addition, supraannular leaflet function provides very low gradients even in small anatomies (17) and the pericardial skirt in the new generation neo 2 design seals effectively against PVL. The transfemoral delivery system has a $18 \mathrm{Fr}$ outer diameter shaft. In detail, first the upper crown is opened, which guarantees stable positioning and supraannular anchoring of the valve. Then the flexible stabilization arches are opened, responsible for the self-aligning properties of the valve, thereby ensuring coaxial alignment. Finally, in step two the lower crown is deployed anchoring the device inside the annulus. The THV is available in three sizes for aortic annulus sizes from 20.0 to $26.3 \mathrm{~mm}$ and are labeled small (S: for aortic annulus sizes 20.0-22.4 mm), medium (M: for aortic annulus sizes $22.5-24.3 \mathrm{~mm}$ ) and large (L: for aortic annulus sizes $24.4-26.3 \mathrm{~mm})(16,18)$ in AS patients. 


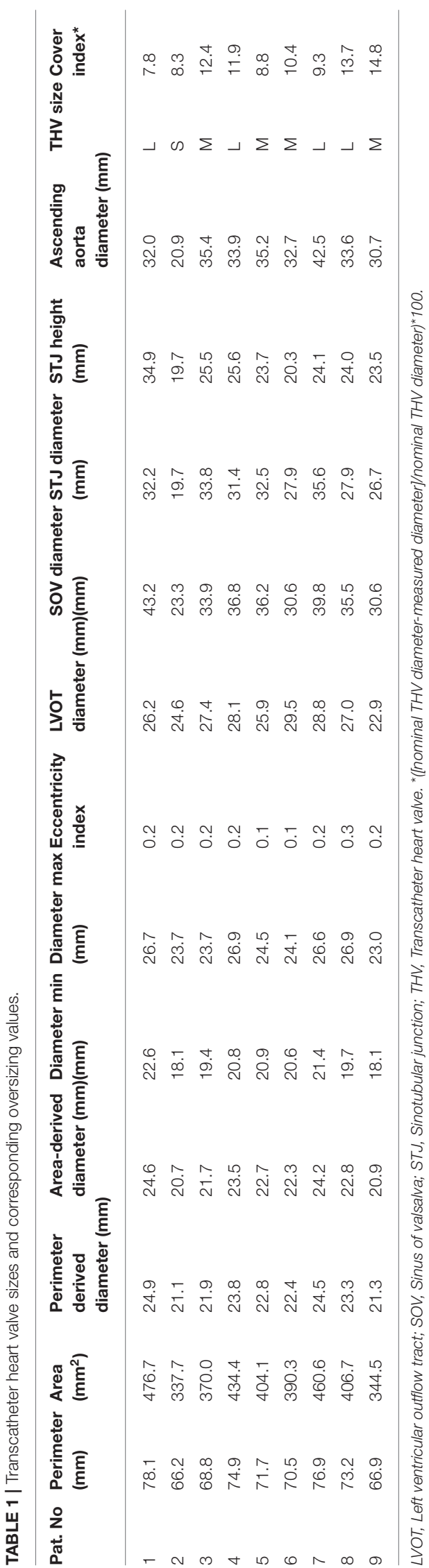

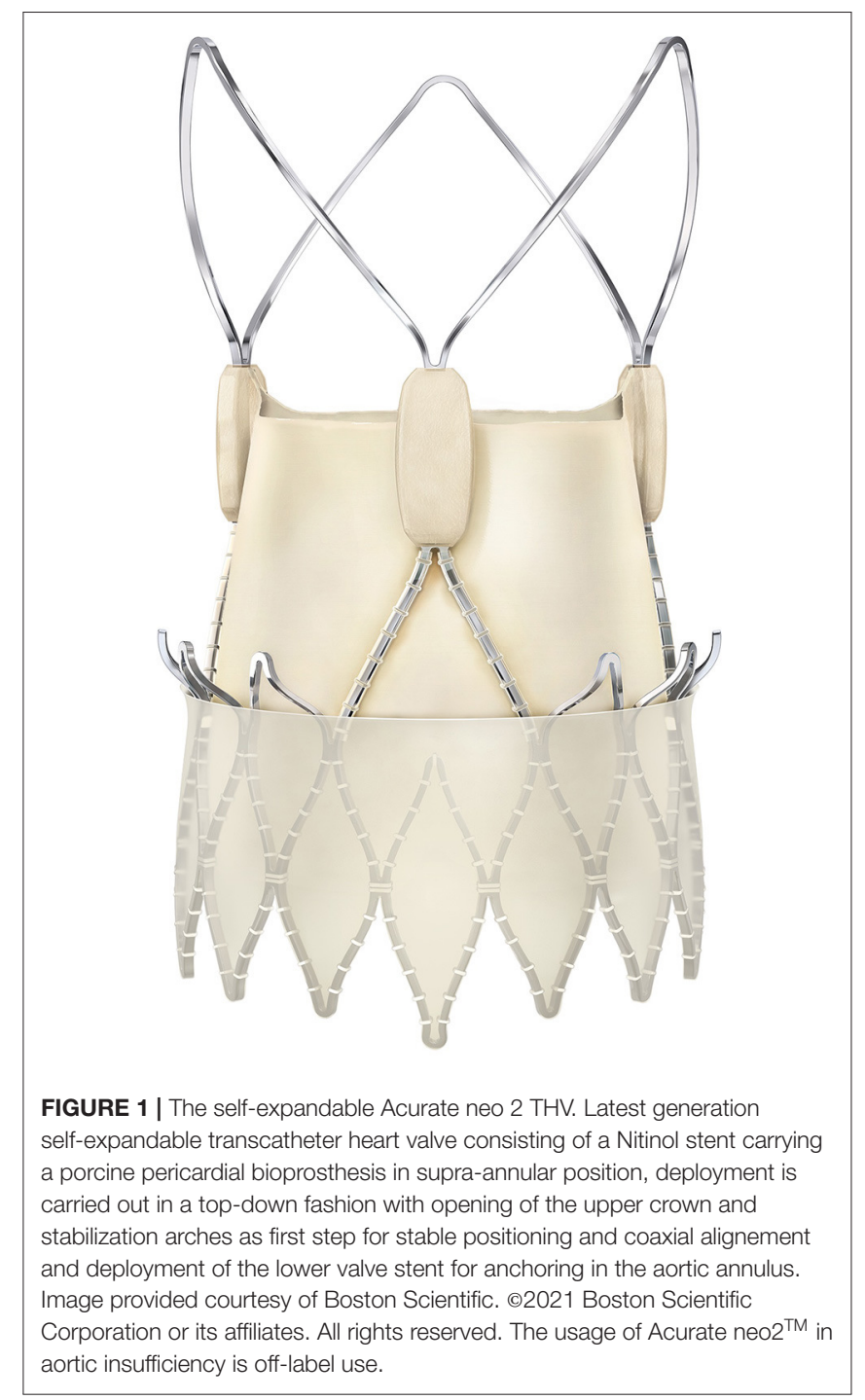

\section{Statistics}

Baseline, intraprocedural and acute follow-up data up to 30 days were retrospectively collected and entered into a standardized database and analyzed. Clinical endpoints were adjudicated in accordance with the updated standardized VARC-2 definitions (19). Data are presented as absolute numbers and percentages for categorical variables and mean values and standard deviation for continuous variables.

\section{RESULTS}

\section{Baseline Demographics}

All 9 consecutive patients ( $88.8 \%$ female, $74.4 \pm 7.1$ years) demonstrated an increased risk profile as reflected by common risk stratification tools (EuroSCORE II $5.5 \pm 3.6 \%$, STS PROM $6.2 \pm 3.0 \%)$. Patients presented with a high comorbidity burden, including 3/9 (33.3\%) with previous sternotomy, 3/9 (33.3\%) patients with concomitant coronary artery disease, and 2/9 
TABLE 2 | Baseline data.

\begin{tabular}{lc}
\hline & Study group $(\boldsymbol{n}=\mathbf{9})$ \\
\hline Age, years & $74.4 \pm 7.1$ \\
Female gender, \% (n) & $88.8(8)$ \\
BMI, kg/m² & $25.1 \pm 5.9$ \\
EuroSCORE II, \% & $5.5 \pm 3.6$ \\
STS PROM Score, \% & $6.2 \pm 3.0$ \\
Diabetes, \% ( $)$ & $22.2(2)$ \\
Arterial hypertension, \% (n) & $55.5(5)$ \\
Previous stroke, \% (n) & $11.1(1)$ \\
Coronary artery disease, \% (n) & $33.3(3)$ \\
Previous sternotomy, \% (n) & $33.3(3)$ \\
S/p LVAD implantation, \% (n) & $11.1(1)$ \\
Extracardiac atheropathy ${ }^{\infty}, \%(n)$ & $11.1(1)$ \\
Arrhythmia, \% (n) & $55.5(5)$ \\
COPD $\infty$ Gold II, \% $(n)$ & $33.3(3)$ \\
Creatinine, mg/dl & $2.0 \pm 2.3$ \\
NYHA $\geq$ III, \% (n) & $77.7(7)$ \\
LVEF, > 50\% & $44.4(4)$ \\
LVEF, 50-30\% & $33.3(3)$ \\
LVEF, > 30\% & $22.2(2)$ \\
\hline
\end{tabular}

BMI, Body mass index; logEuroSCORE, Logistic european system for cardiac operative risk evaluation; STS-PROM, Society of thoracic surgeons predicted risk of mortality; LVAD, Left ventricular assist device; COPD, Chronic obstructive pulmonary disease; NYHA, New York Heart Association functional class; $\infty$ extracardiac atheropathy; $\infty$ COPD according to EuroSCORE definitions.

(22.2\%) patients with diabetes mellitus. $7 / 9$ patients $(77.7 \%)$ of the herein investigated patients were highly symptomatic with a New York Heart Association functional class $\geq$ III. Baseline left ventricular ejection fraction was preserved in $44.4 \%$ (4/9), moderately reduced in $33.3 \%$ (3/9), and severely reduced in $22.2 \%(2 / 9)$. One patient suffered from end-stage heart failure and s/p left ventricular assist device (LVAD) implantation. Detailed patient demographics are summarized in Table 2.

\section{Periprocedural Data}

Procedure time, fluoroscopy time, and volume of contrast agent used were $70.9 \pm 32.4 \mathrm{~min}, 23.2 \pm 13.8 \mathrm{~min}$, and $212.3 \pm$ $105.8 \mathrm{ml}$, respectively. The initial positioning of the THV differed from the standard procedure for AS. The THV was positioned $\sim 1 \mathrm{~mm}$ higher in the aortic annulus compared to interventions for AS. For final deployment (step two), rapid pacing was used for stable THV release. For implantation in the LVAD patient, LVAD flow was reduced prior to valve deployment.

The majority of the patients was treated under local anesthesia and/or analgosedation $(8 / 9,88.8 \%)$. Detailed periprocedural data are summarized in Table 3.

\section{Clinical and Echocardiographic Outcome Data}

All-cause 30-day mortality was 0\% (0/9). Device success and early safety were $100 \%(0 / 9)$ and $77.7 \%$ (7/9), the latter due to two cases
TABLE 3 | Periprocedural data.

\begin{tabular}{lc}
\hline & Study group $(\boldsymbol{n}=\mathbf{9})$ \\
\hline Severe aortic regurgitation, \% $(n)$ & $100(9)$ \\
Baseline peak gradient, $\mathrm{mmHg}$ & $21.3 \pm 12.2$ \\
Baseline mean gradient, $\mathrm{mmHg}$ & $9.9 \pm 5.7$ \\
Invasive pre-implant peak gradient, $\mathrm{mmHg}$ & $4.4 \pm 3.5$ \\
Invasive pre-implant mean gradient, $\mathrm{mmHg}$ & $9.4 \pm 7.4$ \\
Acurate neo, \% ( $n$ ) & $11.1(1)$ \\
Acurate neo 2, \% $(n)$ & $88.9(8)$ \\
Procedure time, min & $70.9 \pm 32.4$ \\
Fluoroscopy time, min & $23.2 \pm 13.8$ \\
Contrast agent, ml & $212.3 \pm 105.8$ \\
Predilatation, \% $(n)$ & $0(0)$ \\
Postdilatation, \% ( $n$ ) & $0(0)$ \\
Anesthesia, \% $(\boldsymbol{n})$ & \\
General anesthesia & $11.1(1)$ \\
Local anesthesia/conscious sedation & $88.8(8)$ \\
Invasive post-implant peak gradient, $\mathrm{mmHg}$ & $2.3 \pm 2.7$ \\
Invasive post-implant mean gradient, $\mathrm{mmHg}$ & $11.7 \pm 7.1$
\end{tabular}

of acute kidney. The VARC-2 adjudicated clinical endpointsstroke, myocardial infarction, or access site complication-did not occur. No postprocedural conduction disturbance or PPM implantation was observed. Intensive care unit and hospital stay were $1.7 \pm 1.1$ and $12.9 \pm 8.8$ days, respectively. Prolonged hospital stay was due to extensive preoperative diagnostic workup because of planned off-label procedures. Echocardiography at 30 days revealed transvalvular peak/mean pressure gradients of $15.3 \pm 12.3 / 7.2 \pm 5.5 \mathrm{mmHg}$. PVL was $\leq$ trace in eight patients $(77.7 \%, 7 / 9)$ and mild in two patients $(22.2 \%, 2 / 9)$.

For detailed outcome parameter see Table 4.

\section{DISCUSSION}

\section{Main Findings}

Main findings of the herein conducted series using the Acurate neo or neo2 THV in TF TAVI for treatment of pure noncalcified AR are (I) the utilized device presents encouraging results in treatment of $\mathrm{AR}$ in patients not eligible for surgery, (II) clinical and echocardiographic results with no documented device migration/embolization and no PVL $>$ mild suggest advantages of the particular design features of the THV for treatment of AR and (III) device success rate suggests that the herein recommended valve sizing algorithm may be especially appropriate for the Acurate neo 2 valve for this special subset of patients.

Over the last years, an increasing number of TAVI procedures for treatment of AR has been documented. Since prevalence of AR increases with age and physicians gather more experience with interventional treatment of this specific subset of patients, this trend is expected to continue. However, TAVI for AR represents certain pre- and intraprocedural challenges which are reflected by documented learning curves and uncertainty 
TABLE 4 | Clinical outcome and echocardiographic results at 30 days.

\begin{tabular}{lc}
\hline & Study group $(\boldsymbol{n}=\mathbf{9})$ \\
\hline All-cause mortality (30 days), \% ( $n)$ & $0(0)$ \\
Stroke (any), \% ( $n)$ & $0(0)$ \\
Myocardial infarction, \% ( $)$ ( $)$ & $0(0)$ \\
Bleeding (major/life threatening), \% ( $)$ & $0(0)$ \\
Access site complications (major), \% (n) & $0.0(0)$ \\
Acute kidney injury (AKIN ${ }^{\star}$ 2, 3), \% (n) & $22.2(2)$ \\
PPM implantation, \% ( $n)$ & $0(0)$ \\
Device success ${ }^{\dagger}, \%(n)$ & $100(9)$ \\
Early safety ${ }^{\ddagger}, \%(n)$ & $77.7(7)$ \\
Intensive care unit stay, days & $1.7 \pm 1.1$ \\
In hospital stay, days & $12.9 \pm 8.8$ \\
Peak gradient, mmHg & $15.3 \pm 12.3$ \\
Mean gradient, mmHg & $7.2 \pm 5.5$ \\
Mild PVL, \% $(n)$ & $22.2(2)$ \\
PVL > mild, \% $(n)$ & $0(0)$
\end{tabular}

PPM, Permanent pacemaker; PVL, Paravalvular leakage; "AKIN, Acute Kidney Injury Network; VARC-2 definitions: ${ }^{\dagger}$ Device success: absence of procedural mortality, correct positioning of a single prosthetic heart valve into the proper anatomical position, intended performance of the prosthetic heart valve (no prosthesis-patient mismatch and mean aortic valve gradient $<20 \mathrm{mmHg}$ or peak velocity $<3 \mathrm{~m} / \mathrm{s}$ and no moderate or severe prosthetic valve regurgitation), ${ }^{\ddagger}$ Early safety at 30 days: all-cause mortality (at 30 days), all stroke (disabling and non-disabling), life-threatening bleeding, acute kidney injury stage 2 or 3 (including renal replacement therapy), coronary artery obstruction requiring intervention, major vascular complication, valve-related dysfunction requiring repeat procedure (Balloon aortic valvuloplasty, TAVI, or SAVR).

regarding adequate valve selection and sizing algorithms (20). For the most frequently used THV systems for treatment of AR varying clinical outcomes and valve sizing algorithms are described. A review of 31 published manuscripts by Yousef et al. (21) evaluating a variety of THV (CoreValve, JenaValve, Direct Flow, Acurate TA, J-Valve, Sapien, Lotus) showed unfavorable clinical outcomes with high 30-day mortality (9.6\%), high intraprocedural need for a second THV (11.3\%), high PPM rate $(10.7 \%)$, and a high incidence of more than moderate PVL $(17.7 \%)$. Although different valve types were combined in this review, oversizing was $\leq 10 \%$ in two thirds $(66.4 \%)$ of cases (21). However, with latest generation devices and increased experience improved outcomes were documented over the last few years. Registry data shows that the most frequently used THV for treatment of AR is the SE CoreValve Evolut/EvolutR (14). Although documented outcomes with this THV generation are significantly improved compared to the initial CoreValve system, mortality, PPM, and residual significant PVL rates are still remarkable with 9,20 , and $6.2 \%$ respectively (12). A series of $24 \mathrm{AR}$ patients who received the Acurate neo THV achieved a device success rate of $87.5 \%$, with moderate PVL in two patients, both when valve oversizing was $>10 \%$. Furthermore, mortality was $4.1 \%$ and PPM rate $21.1 \%$ (13). These reports and our herein described data, although comprising only a small series of patients, suggest that especially the Acurate neo2 THV may be particularly suitable for treatment of AR. Nevertheless, those cited previous reports presented significant higher rates of residual PVL and need for a second THV as compared to implantation of this THV for treatment of AS. Reasons for absence of valve migration/embolization and no PVL $>$ mild in our series may be a combination of a modified sizing algorithm with an oversizing ratio of $>10 \%$ in the majority of patients, the positioning $\sim 1 \mathrm{~mm}$ higher in the aortic annulus than in interventions for AS, the $\mathrm{x}$-shaped stent frame preventing distal or proximal migration as well as extensive user experience with this type of THV. However, it has to be emphasized that the Acurate neo THV presented unfavorable results regarding residual significant PVL compared to a latest generation $\mathrm{BE}$ THV and inferiority regarding mortality and residual significant PVL compared to a latest generation SE THV in randomized controlled trials investigating TAVI in patients with AS $(22,23)$. Why these outcomes differ from the herein seen hemodynamic results remains speculative but may be founded in the valve sizing algorithm and the high implantation height. Reasons for the two cases of postoperative acute kidney injury remain speculative, but are highly likely attributable to preoperative existing reduced kidney function as reflected by preoperative creatinine values and preoperative reduced left ventricular function in the majority of the herein investigated patients.

Of note, in this AR patient cohort, the second deployment step was conducted under RVP to avoid THV dislocation. In one patient with status post LVAD implantation, LVAD flow was briefly reduced to a minimum during device deployment as an additional measure to avoid proximal embolization. The herein described more pronounced oversizing algorithm did not lead to conduction disturbances or postprocedural PPM implantation. This suggests that the documented high PPM rates with other THV systems may rather result from deep implantation height rather than oversizing alone. However, current THV systems are still used off-label and anchoring in non-calcified aortic annuli carries a certain risk for device migration. Therefore, for ideal results and patient safety, THV systems with modified anchoring mechanisms, like the only recently approved JenaValve with clipping of aortic valve cusps, might be advantageous (10).

These results will have to be confirmed in larger patient numbers for further clinical evaluation.

\section{Study Limitations}

Limitations are inherent in a single-center study design with limited patient numbers: patients were not randomized to a specific treatment or THV, therefore patient preselection with hidden confounders may apply. Furthermore, this is a purely descriptive study and conclusions regarding feasibility and especially regarding long-term safety should be drawn with caution.

\section{CONCLUSIONS}

In this limited series of TAVI using the Acurate neo THV for pure non-calcified AR, encouraging results were demonstrated. Thirty-day mortality as well as PPM implantation and significant PVL rates were $0 \%$ in this high-risk patient cohort. Whether these auspicious results are applicable in larger patient cohorts has yet 
to be confirmed since PPM and PVL rates differ significantly compared to AS patients provided with this particular THV.

\section{DATA AVAILABILITY STATEMENT}

The raw data supporting the conclusions of this article will be made available by the authors, without undue reservation.

\section{ETHICS STATEMENT}

The studies involving human participants were reviewed and approved by UKE Ethics Committee. The patients/participants provided their written informed consent to participate in this study.

\section{REFERENCES}

1. Smith CR, Leon MB, Mack MJ, Miller DC, Moses JW, Svensson LG, et al. Transcatheter versus surgical aortic-valve replacement in high-risk patients. N Engl J Med. (2011) 364:2187-98. doi: 10.1056/NEJMoa1103510

2. Reardon MJ, Van Mieghem NM, Popma JJ, Kleiman NS, Søndergaard L, Mumtaz M, et al. Surgical or transcatheter aortic-valve replacement in intermediate-risk patients. $N$ Engl J Med. (2017) 376:1321-31. doi: 10.1056/NEJMoa1700456

3. Leon MB, Smith CR, Mack MJ, Makkar RR, Svensson LG, Kodali SK, et al. Transcatheter or surgical aortic-valve replacement in intermediate-risk patients. N Engl J Med. (2016) 374:1609-20. doi: 10.1056/NEJMoa1514616

4. Baumgartner H, Falk V, Bax JJ, De Bonis M, Hamm C, Holm PJ, et al. 2017 ESC/EACTS Guidelines for the management of valvular heart disease. Eur Heart J. (2017) 38:2739-91. doi: 10.1093/eurheartj/ehx391

5. Otto CM, Nishimura RA, Bonow RO, Carabello BA, Erwin JP III, Gentile F, et al. 2020 ACC/AHA guideline for the management of patients with valvular heart disease: executive summary: a report of the American College of Cardiology/American Heart Association joint committee on clinical practice guidelines. Circulation. (2021) 143:e3571. doi: 10.1161/CIR.0000000000000932

6. Wendler O, Schymik G, Treede H, Baumgartner H, Dumonteil $\mathrm{N}$, Ihlberg L, et al. SOURCE 3 registry: design and 30-day results of the European postapproval registry of the latest generation of the SAPIEN 3 transcatheter heart valve. Circulation. (2017) 135:1123-32. doi: 10.1161/CIRCULATIONAHA.116.025103

7. Grube E, Van Mieghem NM, Bleiziffer S, Modine T, Bosmans J, Manoharan $\mathrm{G}$, et al. Clinical outcomes with a repositionable self-expanding transcatheter aortic valve prosthesis: the international FORWARD study. J Am Coll Cardiol. (2017) 70:845-53. doi: 10.1016/j.jacc.2017.06.045

8. Schaefer A, Schofer N, Goßling A, Seiffert M, Schirmer J, Deuschl F, et al. Transcatheter aortic valve implantation versus surgical aortic valve replacement in low-risk patients: a propensity score-matched analysis. Eur J Cardiothorac Surg. (2019) 56:1131-9. doi: 10.1093/ejcts/ezz245

9. Thielmann M, Tsagakis K, El Gabry M, Jakob H, Wendt D. Transcatheter aortic valve implantation (TAVI) in patients with aortic regurgitation. Ann Cardiothorac Surg. (2017) 6:558-60. doi: 10.21037/acs.2017.09.16

10. Treede H, Rastan A, Ferrari M, Ensminger S, Figulla HR, Mohr FW. JenaValve. EuroIntervention. (2012) 8(Suppl Q):Q8893. doi: 10.4244/EIJV8SQA16

11. Arias EA, Bhan A, Lim ZY, Mullen M. TAVI for pure native aortic regurgitation: are we there yet? Interv Cardiol. (2019) 14:26-30. doi: 10.15420/icr.2018.37.1

12. Anwaruddin S, Desai ND, Szeto WY, Hermiller JB Jr, Sorajja P, Kodali S, et al. Self-expanding valve system for treatment of native aortic regurgitation by transcatheter aortic valve implantation (from the STS/ACC TVT Registry). Am J Cardiol. (2019) 124:781-8. doi: 10.1016/j.amjcard.2019.05.045

\section{AUTHOR CONTRIBUTIONS}

YS made substantial contributions to the conception and design of the work, the acquisition, analysis, interpretation of data for the work, and wrote the manuscript. OB made substantial contributions for the acquisition and analysis of the data. MS, AS, NS, SP, DW, SB, and HR were revising it critically for important intellectual content and made final approval of the version to be published. LC made substantial contributions to the conception and design of the work and the acquisition, analysis, interpretation of data for the work, and he was revising it critically for important intellectual content and made final approval of the version to be published. All authors contributed to the article and approved the submitted version.

13. Purita PAM, Tahoces LS, Fraccaro C, Nai Fovino L, Kim WK, EspadaGuerreiro C, et al. Transcatheter treatment of native aortic valve regurgitation: results from an international registry using the transfemoral ACURATE neo valve. Int J Cardiol Heart Vasc. (2020) 27:100480. doi: 10.1016/j.ijcha.2020. 100480

14. Yoon SH, Schmidt T, Bleiziffer S, Schofer N, Fiorina C, Munoz-Garcia AJ, et al. Transcatheter aortic valve replacement in pure native aortic valve regurgitation. J Am Coll Cardiol. (2017) 70:2752-63. doi: 10.1016/j.jacc.2017. 10.006

15. Schaefer A, Linder M, Treede H, Deuschl F, Schofer N, Seiffert M, et al. Applicability of next generation balloon-expandable transcatheter heart valves in aortic annuli exceeding formally approved dimensions. Clin Res Cardiol. (2016) 105:585-91. doi: 10.1007/s00392-0150954-9

16. Kim WK, Hengstenberg C, Hilker M, Schäfer U, Rudolph TK, Toggweiler $\mathrm{S}$, et al. CollaboratorsTranscatheter aortic valve implantation with the ACURATE neo valve: indications, procedural aspects and clinical outcomes. EuroIntervention. (2020) 15:e1571-79. doi: 10.4244/EIJ-D-19H00908

17. Mauri V, Kim WK, Abumayyaleh M, Walther T, Moellmann H, Schaefer $\mathrm{U}$, et al. Short-term outcome and hemodynamic performance of nextgeneration self-expanding versus balloon-expandable transcatheter aortic valves in patients with small aortic annulus: a multicenter propensity-matched comparison. Circ Cardiovasc Interv. (2017) 10:e005013. doi: 10.1161/CIRCINTERVENTIONS.117.005013

18. Schäfer U, Conradi L, Diemert P, Deuschl F, Schofer N, Seiffert M, et al. Symetis ACURATE TAVI: review of the technology, developments and current data with this self-expanding transcatheter heart valve. Minerva Cardioangiol. (2015) 63:359-69.

19. Kappetein AP, Head SJ, Généreux P, Piazza N, van Mieghem $\mathrm{NM}$, Blackstone $\mathrm{EH}$, et al. Updated standardized endpoint definitions for transcatheter aortic valve implantation: the Valve Academic Research Consortium-2 consensus document. I Thorac Cardiovasc Surg. (2013) 145:6-23. doi: 10.1016/j.jtcvs.2012. 09.002

20. Takagi H, Hari Y, Kawai N, Ando T; ALICE (All-Literature Investigation of Cardiovascular Evidence) Group. Meta-analysis and meta-regression of transcatheter aortic valve implantation for pure native aortic regurgitation. Heart Lung Circ. (2020) 29:729-41. doi: 10.1016/j.hlc.2019. 04.012

21. Yousef A, MacDonald Z, Simard T, Russo JJ, Feder J, Froeschl MV, et al. Transcatheter Aortic Valve Implantation (TAVI) for native aortic valve regurgitation - a systematic review. Circ J. (2018) 82:895-902. doi: 10.1253/circj.CJ-17-0672

22. Lanz J, Kim WK, Walther T, Burgdorf C, Möllmann H, Linke A, et al. Safety and efficacy of a self-expanding versus a balloon-expandable bioprosthesis for transcatheter aortic valve replacement in patients with symptomatic severe 
aortic stenosis: a randomised non-inferiority trial. Lancet. (2019) 394:161928. doi: 10.1016/S0140-6736(19)32220-2

23. Tamburino C, Bleiziffer S, Thiele H, Scholtz S, Hildick-Smith D, Cunnington $\mathrm{M}$, et al. Comparison of self-expanding bioprostheses for transcatheter aortic valve replacement in patients with symptomatic severe aortic stenosis: SCOPE 2 randomized clinical trial. Circulation. (2020) 142:243142. doi: 10.1161/CIRCULATIONAHA.120.051547

Conflict of Interest: MS reports lecture fees and travel expenses from Boston Scientific. LC is advisory board member for Boston Scientific.

The remaining authors declare that the research was conducted in the absence of any commercial or financial relationships that could be construed as a potential conflict of interest.
Publisher's Note: All claims expressed in this article are solely those of the authors and do not necessarily represent those of their affiliated organizations, or those of the publisher, the editors and the reviewers. Any product that may be evaluated in this article, or claim that may be made by its manufacturer, is not guaranteed or endorsed by the publisher.

Copyright (c) 2022 Schneeberger, Seiffert, Schaefer, Bhadra, Schofer, Pecha, Westermann, Blankenberg, Reichenspurner and Conradi. This is an open-access article distributed under the terms of the Creative Commons Attribution License (CC $B Y)$. The use, distribution or reproduction in other forums is permitted, provided the original author(s) and the copyright owner(s) are credited and that the original publication in this journal is cited, in accordance with accepted academic practice. No use, distribution or reproduction is permitted which does not comply with these terms. 